\title{
NECESIDADES DE LA INDUSTRIA DE MADERA ASERRADA EN CHILE
}

\author{
Sergio Vidaurre Echeverría*
}

\section{RESUMEN}

En el presente artículo se identifican y discuten los principales problemas y necesidades que en la actualidad presenta la industria nacional de la madera aserrada, entregando también conclusiones y recomendaciones generales.

El trabajo se elaboró mediante una revisión de la bibliografía pertinente, entrevistas a diversos expertos en la materia y análisis de la información obtenida por profesionales del Instituto Forestal (INFOR) a través de encuestas efectuadas a todos los aserraderos del pais, con el apoyo de la experiencia recogida en visitas técnicas a algunas plantas representativas.

Se aprecia que esta industria presenta problemas importantes que resolver a todo nivel. Dichos problemas se relacionan con: materia prima; variables del proceso de producción; uso, mercado y normalización del producto; necesidades de mantención de las herramientas de corte; capacitación de personal y necesidades de maquinaria y equipos. Además, se estima que hay una carencia de investigación básica y aplicada con respecto al aserrío de las especies de interés comercial que crecen en Chile.

\section{ABSTRACT}

This article presents and discusses actual problems and requirements that are considered relevant for the national lumber industry, also giving general conclusions and recommendations.

This work involved a literature review, interviewing experts and analyzing the information gathered by the Instituto Forestal (INFOR) staff through inquests to all the Chilean sawmills and by on-site visits to some representative plants.

There are many important problems related to the industry that need to be solved. These problems concern: raw material; production process variables; uses, marketing and standardization of the product; equipment maintenance; training needs and machinery investments. It is also pointed out that there is a lack of basic and applied research in relation to sawing the commercial timber species grown in Chile.

* Ingeniero Forestal, U. de Chile Master of Forestry, University of Washington División Regional (Area Industrias) Barros Arana № 121, 3er. piso Conccpción. 


\section{INTRODUCCION}

La importancia creciente que ha ido adquiriendo la industria nacional de madera aserrada, especialmente la de Pino radiata, se refleja en la instalación, durante la última década, de siete aserraderos mecanizados con capacidades individuales de producción muy por encima de $50.000 \mathrm{~m}^{3} /$ año. Por otra parte, se menciona que para fines de siglo será necesario contar al menos con otros diez aserraderos con una capacidad promedio del orden de $100.000 \mathrm{~m}^{3} / \mathrm{año(}(1)$.

Debido a la relevancia de este sector industrial, resulta evidente la necesidad de planificar con el fin de identificar los problemas y necesidades más urgentes que se requiere abordar con respecto a esta industria y de sugerir líneas globales de acción para los próximos años. Con esa base, se supone que a futuro se podrán ir concretando proyectos más específicos en cada una de dichas lineas.

En el presente artículo se describen problemas y necesidades y se entregan sugerencias ligadas tanto con el producto como con el proceso de producción de esta industria, abarcando desde la materia prima hasta el producto final. Además, este artículo intenta orientar en parte la investigación en este campo $\mathrm{y}$, en general, discute temas que se consideran relevantes para ir mejorando el desarrollo de la industria de aserrio en Chile.

\section{ANTECEDENTES GENERALES DE LA INDUSTRIA DE ASERRIO EN CHILE}

De acuerdo con el censo realizado por INFOR (10), en 1985 existían en Chile aproximadamente 1597 aserraderos, los cuales produjeron cerca de $2.200 .000 \mathrm{~m}^{3}$ (13). La capacidad total de producción a un turno de esta industria es aproximada a $4.400 .000 \mathrm{~m}^{3} / \mathrm{año}$, de modo que sólo se aprovecha alrededor de un $50^{\circ} \%$ de esa capacidad $(9,10,13,14)$. Como se explicará más adelante, existen notorias diferencias en la utilización de la capacidad instalada según el tamaño de los aserraderos.

Esta industria nacional se caracteriza por una alta atomización en el número de aserraderos, propietarios y localización geográfica, como así mismo por la heterogeneidad del tamaño de las plantas y del tipo y calidad de la tecnología empleada. La gran mayoría de los establecimientos del pais, al menos un $80 \%$ o, son de tipo móvil o temporal; el resto corresponde a aserraderos permanentes.

El número de aserraderos según su tamaño, nivel de producción e importancia en la producción total, se presenta en el Cuadro 1 .

En el cuadro puede advertirse que la producción está muy concentrada en pocos aserraderos. En 1985, los 22 establecimientos de mayor tamaño produjeron un poco más del $35 \%$ del total nacional; en 1984, los 24 aserraderos principales dieron origen a alrededor del $50 \%$ del total (9). A su vez, en 1984, las 20 plantas más grandes exportaron el $72 \%$ del volumen colocado en el exterior (10).

La localización de las plantas y la importancia relativa de las diferentes regiones de Chile en el volumen producido de madera aserrada se indica en el Cuadro 2.

En la VIII Región se origina más del $50 \%$ de la producción nacional de madera aserrada. Otras regiones de importancia son la IX, la VII y también la X; que cuenta con el mayor número de aserraderos en el pais, pero en general pequeños y subutilizados. La producción de todas las otras regiones suma sólo un $7 \%$ del total; sin embargo, es de interés mencionar que entre 1984 y 1985 se registró en las Regiones IV y XI un significativo crecimiento relativo de la producción, que llegó a un $182 \%$ y un $75 \%$ o respectivamente $(13,14)$.

Además, la VIII Región cuenta con la gran mayoria de los aserraderos más grandes y de mejor tecnología que operan en el país. En esta zona están cerca del $86 \%$ de los establecimientos grandes (que producen más de $18.000 \mathrm{~m}^{3} /$ año) y el $73 \%$ o de los medianos (que producen entre 10.000 y $\left.17.999 \mathrm{~m}^{3} / \mathrm{año}\right)(10,14)$. Por otro lado, los modernos aserraderos mecani- 


\section{CUADRO 1}

NUMERO DE ASERRADEROS Y PRODUCCION, SEGUN TAMAÑO

\begin{tabular}{lcrc}
\hline Tamaño (capacidad m3/año) & $\begin{array}{c}\mathbf{N}^{\mathbf{0}} \text { de aserraderos } \\
\text { Cantidad }\end{array}$ & \multicolumn{1}{c}{ \%o } & $\begin{array}{c}\text { Participación en la } \\
\text { producción de 1985 } \mathbf{( \%} / \mathbf{0})\end{array}$ \\
\hline Grandes $(>18.000)$ & 14 & 0,9 & 28,6 \\
Medianos $(10.000-17.999)$ & 8 & 0,5 & 6,8 \\
Chicos $(1.000-9.999)$ & 332 & 20,8 & 48,7 \\
Pequeños $(<1.000)$ & 1.243 & 77,8 & 15,9 \\
Total & $\mathbf{1 . 5 9 7}$ & $\mathbf{1 0 0 , 0}$ & $\mathbf{1 0 0 , 0}$ \\
\hline
\end{tabular}

FUENTES: INFOR 1985, 1986.

zados con capacidad de producción a $50.000 \mathrm{~m}^{3}$ /año se localizan también en la VIII Región, salvo uno, el Aserradero Copihue, que se ubica en la VII Región. Como resultado de su gran importancia, la VIII Región es actualmente el origen de casi toda la madera aserrada que se exporta y cuenta además con todos los puertos de que el pais dispone para este objetivo. Por último, la VIII Región basa su potencialidad casi enteramente en las plantaciones de Pinus radiata $\mathrm{D}$. Don.

Al alejarse de la VIII Región, los establecimientos tienden a ser chicos o muy pequeños y presentan además capacidades de producción muy subutilizadas. De la Región IX al sur, existe abundancia de aserraderos móviles o de montaña, con proceso de aserrío simple y sierra principal circular de diente postizo, los cuales procesan básicamente maderas nativas. En la IX Región, por ejemplo, a principios de 1985 existían 317 plantas, de las cuales 285 eran móviles o de montaña. En las regiones extremas (IV, XI y XII), todos los aserraderos son muy pequeños (con capacidad inferior a $1.000 \mathrm{~m}^{3}$ /año), con excepción de sólo un establecimiento en la XII Región $(10,14)$.

En cuanto a las especies actualmente empleadas, es evidente que la industria nacional de madera aserrada basa su producción en el Pino radiata, que alcanza a un $85 \%$ o del total. El

CUADRO 2

NUMERO DE ASERRADEROS Y PRODUCCION POR REGION

\begin{tabular}{lrrr}
\hline Región & $\mathbf{N}^{\mathbf{0}}$ aserraderos & Producción $\mathbf{1 9 8 5}\left(\mathbf{m}^{\mathbf{3}}\right)$ & Participación $(\mathbf{\%} / \mathbf{0})$ \\
\hline IV & 6 & 1.234 & 0,1 \\
V & 17 & 39.251 & 1,8 \\
VI & 46 & 73.532 & 3,4 \\
VII & 98 & 293.612 & 13,4 \\
VIII & 485 & 1.176 .452 & 53,7 \\
IX & 317 & 308.785 & 14,1 \\
X & 561 & 260.608 & 11,9 \\
XI & 28 & 14.495 & 0,7 \\
XII & 44 & 22.660 & 1,0 \\
\hline
\end{tabular}

FUENTES: INFOR 1985, 1986. 
resto del producto proviene de diversas especies nativas y exóticas, las cuales antiguamente representaban casi toda la producción. Algunas de estas especies utilizadas en la industria son: Laurelia philippiana (Phil.) Losser (Tepa), Nothofagus dombeyi (Mirb.) Blume (Coihue), Nothofagus obliqua (Mirb.) Oerts. (Roble), Nothofagus pumilio (Poepp et Endl.) Krasser. (Lenga), Populus spp. (Alamo), Eucalyptus spp. (Eucalipto), Nothofagus alpina (Poepp et Endl.) Oerst. (Rauli) y Eucryphia cordifolia Cav. (Ulmo).

\section{CALIDAD Y USO DE LA MADERA ASERRADA EN CHILE}

El análisis de la calidad y el uso de la madera aserrada actualmente producida en el país se enfoca en torno a distintos aspectos:

- Calidad de la materia prima (trozos).

- Proceso de corte y mantención de sierras.

- Necesidades de capacitar mano de obra.

- Tratamientos posteriores al aserrío (baño antimancha, secado e impregnación).

- Problemas especiales asociados a los pequeños aserraderos.

- Problemas en el uso de la madera aserrada.

- Necesidad e importancia de normalizar la madera aserrada en Chile.

a) Calidad de los trozos. El manejo del bosque y las prácticas silviculturales (técnicas de plantación, poda y raelo) son de alta relevancia para la calidad de la madera aserrada, debido a que afectan significativamente la calidad de la materia prima (trozos) y, en consecuencia, del producto derivado y su comercialización.

Un problema importante del recurso forestal actual maduro lo constituye el hecho de que la mayor parte de las plantaciones no han sido manejadas ( $\sin$ raleos ni podas), lo que se traduce en una alta proporción de troncos nudosos y de pequeños diámetros, los cuales por lo general son comercializados como trozos para pulpa y papel y para producir madera aserrada habitualmente de calidad inferior o para usos menores. El hecho de destinar trozos de diámetros menores para madera aserrada trae además como consecuencia una alta proporción de piezas con médula o cercana a ésta, lo que origina defectos de alabeo y de aspecto, que se traducen en un menor valor para el producto.

Sólo recientemente las prácticas silviculturales tendientes a mejorar la calidad de la madera aserrada están siendo implementadas con técnicas relativamente avanzadas en las empresas más grandes. En el país estas prácticas sólo se aplican desde el comienzo de esta década y en alrededor de la mitad de las principales compañías.

Es de gran importancia hacer extensivas estas técnicas a todo el recurso, por su alta incidencia en la calidad y en el mayor precio que se puede obtener del producto, factores que permitirian al país incrementar el rango y la calidad de la producción. Lo ideal sería producir trozos de mayores diámetros (lo que incide en general en un mayor rendimiento del aserradero) $y$ sin nudos (lo que incide en un mayor valor del producto final), para lo cual se requieren raleos y podas tempranas; en este sentido, se aprecian tendencias positivas, que son favorecidas por el costo relativamente bajo de la mano de obra y el rápido crecimiento de las plantaciones.

Además, otros factores importantes en la calidad de la madera, como son su densidad y el ancho de los anillos, pueden ser modificados con el manejo del recurso forestal.

En resumen, todas las medidas ya señaladas serán de alta importancia para obtener madera aserrada de mayor calidad exportable, hecho que implica mejores precios y nuevos mercados. Esta posibilidad de interés, considerando la limitada capacidad actual que posee la demanda interna para absorber los volúmenes producidos.

Finalmente, como materia prima se estima que la madera de Pino radiata no debería tener mayores complicaciones en el proceso de aserrado propiamente tal (5). Esta es una madera blanda (de una densidad básica aproximada entre 0,4 y $0,45 \mathrm{ton} / \mathrm{m}^{3}$ (15) ) y fácil de aserrar, 
que ocasiona un bajo desgaste relativo de los elementos de corte. También es una madera fácil de secar y de impregnar, aunque tiene el inconveniente de que se mancha con facilidad.

b) Proceso de corte y mantención de sierras. Con respecto al proceso de corte de los trozos, se aprecian diversas anomalías: defectos en la terminación superficial de la madera aserrada, escuadrías desuniformes a lo largo de una pieza y variaciones de espesor entre piezas. Estos defectos representan un serio problema en relación a la utilización de este producto en la construcción, por ejemplo, como producto exportable.

En el país se advierten notorias diferencias en la terminación superficial de la madera aserrada, según el tipo de sierras que se emplean en el proceso de producción. En general la peor calidad se obtiene cuando se emplean sierras circulares, debido a su incorrecta preparación: el afilado y tensionado se lleva a cabo habitualmente en forma manual en la mayoría de los aserraderos pequeños.

En la actualidad se estima que más del $50 \%$ de la producción chilena se obtiene con sierra circular (fundamentalmente diente postizo) en la máquina principal (5) y casi un $94 \%$ de los aserraderos tiene sierra principal circular (10), la que al no estar apropiadamente preparada origina, como se dijo, una deficiente terminación superficial del producto, aparte de incrementar aun más la pérdida de materia prima debido al mayor ancho de corte, lográndose rendimientos normalmente inferiores al $45 \%$ o (7). El resto de la producción se obtiene con sierras huinchas y alternativas, las cuales se preparan habitualmente con máquinas automáticas que mejoran la terminación y aprovechamiento, de tal modo que el rendimiento sube hasta cifras del orden de $50 \%$ o. (7).

Finalmente, se pueden mencionar algunas formas por medio de las cuales se estima que se podría reducir en forma significativa los principales problemas actuales orginados por las sierras circulares de diente postizo, en cuanto a la baja calidad del producto y al bajo rendimiento en el proceso. En primer lugar, estas sierras deberian tender en lo posible a realizar sólo el encuadrado del trozo, fabricando por tanto sólo "basas", para posteriormente producir las tablas con sierra huincha. Por otro lado, es importantísimo darle un fuerte énfasis a la mantención de las sierras (en especial un buen tensionado), ya que en la actualidad esta variable está muy descuidada. Si se mejoran estos aspectos, se obtendrán notables mejorías. Por último, podrá ser factible en ciertos casos cambiar la sierra, lo que implicará cambiar todo el equipo que la acompaña; ésta es una solución más cara, pero que podría justificarse cuando la operación general esté en condición muy deficiente o cuando el beneficio esperado supere este costo.

c) Necesidad de capacitar mano de obra. De acuerdo con cifras de INFOR (13), la ocupación forestal total en 1984 fue cercana a 66.000 personas, de las cuales alrededor de un $39 \%$ trabajaban directamente en las industrias forestales y el resto indirectamente en faenas de silvicultura y en servicios forestales. De las 25.250 personas que laboraron en las industrias, 20.740 lo hicieron para las industrias mecánicas de la madera y 4.511 que operan en las plantas de pulpa y papel. Se estima que en estas cifras se incluyen alrededor de 2.500 ingenieros y técnicos y del orden de 1.500 trabajadores especializados $(1,13)$.

En cuanto a la especialización de la mano de obra, se puede afirmar que existe una cantidad suficiente de trabajadores especializados para faenas de silvicultura y explotación, pero una escasez de técnicos para la industria forestal.

Sin embargo, existe la necesidad de entrenar técnicos de alto nivel especialmente para trabajar en las industrias mecánicas de la madera. Respecto al entrenamiento de trabajadores especializados para la industria, el INFOR ha interrumpido los cursos que anteriormente ofrecía. Por otro lado, las grandes compañías ofrecen cursos actualmente, pero en general limitados sólo a pocas personas $(1,5)$.

Concretamente con respecto a la industria del aserrío, se estima que en el país existe un 
déficit de personal calificado. Se requiere capacitar técnicos de alto nivel para trabajar en los aserraderos y en las plantas de elaboración, formar especialistas en afilado y mantención de sierras y también impartir conocimientos teóricos al personal.

d) Tratamientos posteriores al aserrio. Los tratamientos a que se puede someter la madera aserrada son el baño antimancha, la impregnación y el secado.

Baño antimancha. En los aserraderos grandes y medianos del país, a continuación del paso por las sierras despuntadoras, la madera es sometida a un baño antimanacha a base de una solución de pentaclorofenato de sodio y bórax, con el objeto de prevenir temporalmente la mancha azul y la pudrición. En los aserraderos pequeños este baño es poco frecuente.

Impregnación de la madera. Son pocos los aserraderos que cuentan con plantas para la impregnación de la madera aserrada. INFOR (11) ha identificado 12 aserraderos en el país que disponen de plantas impregnadoras. Por otro lado, se estima que en Chile existe un total aproximado de 20 plantas impregnadoras de madera con una capacidad de producción cercana a los $175.000 \mathrm{~m}^{3}$ /año-turno $(6,7)$.

Secado de la madera aserrada. En la mayoría de los aserraderos nacionales el secado se efectúa al aire, de modo que sólo se alcanza el contenido de humedad de equilibrio para cada zona. Un secado apropiado es otro factor reconocidamente decisivo en la calidad de la madera. En este sentido, el contenido de humedad que se logra por el secado al aire es inadecuado para muchos usos de la madera en la construcción.

En relación al secado artificial, en el país existe una clara falta de capacidad, hecho que se agravará en los próximos años por los incrementos esperados de la producción y exportación de madera aserrada. En el presente, se dispone en Chile de aproximadamente 54 plantas de secado de madera aserrada con una capacidad total cercana a $7.000 \mathrm{~m}^{3}$ (2). De éstas, 35 plantas son de secado convencional con una capacidad aproximada de $5.400 \mathrm{~m}^{3}\left(3.070 \mathrm{~m}^{3}\right.$ de Pino radiata y $2.370 \mathrm{~m}^{3}$ para otras especies); 15 son de secado por condensación, vacio y aire forzado, con una capacidad del orden de $830 \mathrm{~m}^{3}$ y 4 plantas son de secado a alta temperatura con capacidad para $740 \mathrm{~m}^{3}$.

Por otra parte, la capaciaad total anual de secado de Pino radiata puede estimarse en $440.000 \mathrm{~m}^{3}(2)$, siendo la capacidad de los secadores convencionales levemente superior a la de los secadores de alta temperatura. Considerando que en 1985 se exportaron cerca de $706.000 \mathrm{~m}^{3}$ de madera aserrada (13), actualmente se tendría la capacidad para secar artificialmente poco más del $60 \%$ de dicha madera, sin considerar la necesidad de secar madera para uso nacional. Por último, el secado artificial se practica en la mayoría de los aserraderos más grandes con capacidades de secado normalmente muy inferiores a las respectivas capacidades de producción de madera aserrada.

El secado artificial de la madera aserrada es actualmente uno de los principales problemas y un desafío para mejorar la calidad y las exportaciones de este producto. En general, son notorias las anomalías con respecto a las técnicas del secado artificial que se aplican en el pais, lo cual repercute obviamente en la calidad de la madera aserrada. Por ejemplo, se cometen errores en el manejo de las variables de secado, existe falta de conocimiento básico del comportamiento de la madera y normalmente se efectúa un deficiente control de calidad del producto final. Estas deficiencias originan habitualmente variaciones excesivas en el contenido de humedad del producto y frecuentes defectos asociados con el proceso de secado; con respecto a estos problemas se debe tener presente que las exigencias del mercado externo son bastante rigurosas. Aún no existe consenso en el país en el sentido de que secar es importante y, por otra parte, los fabricantes extranjeros de secadores normalmente no entregan programas de secado aplicables a especies de interés nacional, como el Pino radiata. 
e) Problemas especiales asociados a los aserraderos pequeños. Los aserraderos pequeños suelen tener un alto grado de subutilización de sus capacidades de producción, se encuentran habitualmente en pobres condiciones técnicas y los problemas relacionados con la calidad del producto son comúnmente mucho más acentuados que los expuestos para los aserraderos de tamaños mayores.

Muchos de estos aserraderos son móviles y operan sólo durante parte del año. En ellos, el período de menor producción o suspensión de las operaciones ocurre en los meses más lluviosos, por problemas de abastecimiento de madera y colocación del producto en los centros de consumo. Esto contrasta con lo que ocurre en los aserraderos de mayor capacidad, los cuales están respaldados por una infraestructura adecuada y suficiente cantidad de materia prima para operar a producciones cercanas a sus capacidades.

Los establecimientos pequeños en general cuentan con maquinaria obsoleta. Las deficiencias de potencia, asi como las imperfecciones de tipo mecánico, impiden lograr un buen proceso de corte y producen por lo general una mala terminación superficial e irregularidades en el espesor de la madera aserrada.

Por otra parte, debido al gran ancho de corte de las sierras circulares empleadas en estas plantas, la utilización de la madera es baja, como se ha dicho antes, produciéndose gran cantidad de desechos. Normalmente no se emplean descortezadores y en muchos casos las "tapas", y posiblemente también otros desechos como cantos y despuntes, son usados como combustible en los locomóviles. Varios de estos aserraderos sólo realizan un aserrado primario produciendo "basas" que son posteriormente reaserradas con otros equipos, como una forma de mejorar la deficiente calidad del producto. Finalmente, estas plantas no disponen de secadores artificiales, con las consiguientes limitaciones en la humedad de la madera aserrada.

Algunos expertos (5) coinciden al afirmar que varios de los aserraderos chicos se están quedando sin trozos, señalando también que el bosque se está concentrando cada vez en menos manos. Ellos estiman que muchos de estos establecimientos pequeños van a tender a desaparecer.

Además, es posible suponer que a medida que la demanda interna vaya aumentando las exigencias de calidad de la madera aserrada, la mayoría de estas plantas más pequeñas estarán obligadas a mejorar, o de otro modo probablemente deberán cerrar. También es probable que una solución para los propietarios de bosques menores sea unirse a fin de construir un aserradero de capacidad mayor, eficiente, con secadores artificiales y apto para producir un producto de calidad.

Por último, es de interés señalar que para que los aserraderos operen en forma económica, cada vez es más importante incorporar los procesos de astillado y descortezado de la madera, especialmente por el alto precio de exportación que están adquiriendo actualmente las astillas. Los aserraderos grandes tienen la ventaja de aprovechar casi todo el material (astillas, corteza, a veces el aserrrín, etc.). En general, estos establecimientos mayores están operando a un buen nivel económico.

f) Problemas en el uso de la madera aserrada. Lamentablemente el aprovechamiento de la madera en el país no guarda relación con los recursos disponibles, los cuales son subutilizados tanto cuantitativa como cualitativamente. En general, existen deficiencias en cuanto a la calidad y utilización eficiente de algunos productos importantes, tales como la madera aserrada y los paneles. Un ejemplo de lo anterior lo constituye el rubro de la construcción en madera, el cual muestra un fuerte retraso tecnológico; de hecho en Chile la madera está en un nivel secundario entre los materiales de construcción, empleándose más bien en situaciones de emergencia, sin mayores exigencias de calidad ni de tratamientos para resistir las condiciones del medio donde se construye. 
Pese a que Chile es un pais forestal y tiene un serio problema de escasez habitacional (cercano a 1.000 .000 de viviendas), paradojalmente existe una reticencia a emplear la madera en la construcción. Este hecho obedece a diversas razones, entre las cuales se puede mencionar: desconocimiento de las bondades de la madera; desconocimiento de su mejor forma de empleo tanto en la construcción como en otros usos; desprestigio de la madera, ya que erróneamente la gente la asocia con un material barato, con problemas de pudrición, manchas y combustión y con diferentes deficiencias de calidad. Por estos motivos, la gran mayoría de los proyectos de viviendas derivan hacia el concreto, hormigón armado, ladrillo o adobe.

Esta situación es radicalmente opuesta en los paises de elevado nivel de desarrollo industrial, tales como los Estados Unidos, Canadá, Japón y muchos países europeos, en los cuales los productos de la madera están bien normalizados, son usados eficientemente, constituyen el material mayoritariamente empleado en la construcción y gozan de un excelente prestigio y aceptación. En los Estados Unidos, por ejemplo, cerca de $80 \%$ de la construcción se realiza con materiales a base de madera.

Las deficiencias de calidad y normalización de la madera aserrada (y también de los paneles) han contribuido significativamente a desprestigiar la madera como material de construcción. El producto que se incorpora al mercado a menudo presenta diversos defectos, como ya se ha expuesto: medidas y escuadrías irregulares y no adecuadas a las necesidades del consumidor, contenidos de humedad muy altos, presencia de manchas, grietas, nudos, etc.

g) Necesidad e importancia de normalizar la madera aserrada en Chile. Se han efectuado en el pais intentos por poner en uso normas de calidad para la madera aserrada, pero con pobres resultados en la práctica. Existen normas generales de clasificación visual por aspecto y últimamente también por resistencia para el Pino radiata, las cuales no se aplican. Por lo tanto, la comercialización en el mercado interno continúa en la mayoría de los casos efectuándose sobre estándares de calidad carentes de objetividad, que productores y consumidores fijan arbitrariamente.

Por otro lado, las ventas al mercado externo han debido adaptarse rigurosamente a las diferentes exigencias impuestas por cada país importador. Podría afirmarse que sólo se diferencia entre madera aserrada para el mercado interno y para la exportación. Para productos a base de maderas nativas, se dispone de una clasificación de calidades según las categorías I, II, III, IV y de desecho, la cual es ambigua en el sistema de clasificación.

En Chile no existen normas de clasificación por resistencia para la madera estructural, con lo cual se hace difícil el uso estructural del Pino radiata en los sistemas constructivos. Además, la madera se emplea en forma muy conservadora en la construcción, en relación a su resistencia, lo que se traduce en un uso ineficiente del material. Es evidente que el obstáculo técnico más importante que frena un uso más amplio de la madera en la construcción es la carencia de reglas apropiadas de calidad y clasificación por resistencia.

En resumen, como una etapa básica tendiente a superar los problemas mencionados, y como una forma de incentivar el uso y las exportaciones de madera aserrada (y también de tableros). es esencial dictar y exigir la aplicación de normas o especificaciones de calidad para el producto. La existencia y acatamiento de normas constituye uno de los factores importantes que se debe considerar si se pretende mejorar técnicamente los aserraderos.

\section{MAQUINARIAS Y EQUIPOS}

En esta parte se exponen las necesidades consideradas de mayor interés relativas a las maquinarias y equipos de aserrío, y los requerimientos que se complementan con los ya mencionados. 
a) Una de las principales necesidades de modernización o reposición de equipos se aprecia en los aserraderos móviles o de sierra circular de dientes postizos. Como se ha dicho, son antiguos y están operando en deficientes condiciones: sierra con gran ancho de corte, mala mantención, carros no alineados, guias en mal estado, problemas de potencia, etc.

En muchos de esos aserraderos será necesario cambiar las sierras para reducir el ancho de corte y hacerlo más exacto. El cambio de sierras obviamente implicará un cambio de los equipos y también de los accesorios, ya que se requieren otras velocidades de operación. Estas modernizaciones aumentarán la calidad del producto, la producción y el rendimiento del proceso.

b) Otra adaptación tecnológica que se estima necesaria, se refiere al hecho de que en los próximos años se contará mayoritariamente con trozos de diámetros menores que los actuales. Esto implica que seguramente se requerirán equipos tales como el astillador canteador (chipper canter) y también sierras circulares dobles, para procesar más eficientemente dicha materia prima.

c) Por otra parte, los expertos opinan que en muchos aserraderos y también en algunas plantas elaboradoras, debería realizarse una modernización en los sistemas de transporte (correas transportadoras, sistemas de rodillos, cadenas, etc.) (5), para lograr un flujo rápido en el transporte de la materia prima y del producto, sin que haya que esperar en muchos casos a un cargador frontal para que solucione alguna obstaculización en el proceso. Anomalías en relación a lo anterior ocurren normalmente en casi todos los aserraderos mecanizados, donde es más crítico cualquier problema de este tipo. Teniendo las anteriores consideraciones en mente. se evitan tiempos muertos en las maquinarias, contribuyendo asi al aprovechamiento máximo de su capacidad.

d) Además, será necesario invertir o reponer equipos para modernizar o mejorar el sistema de extracción de los desechos generados en los procesos de producción.

e) Otras reposiciones de maquinarias o equipos seguramente serán necesarias a consecuencia de una mantención actual deficiente. Es habitual que se efectúe mantención cuando la respectiva máquina o sus piezas fallan. Además, como política general, sería conveniente efectuar una mantención preventiva.

f) También se requerirá reponer, modernizar o invertir en diversos equipos altamente relevantes para estas industrias: secadores, plantas impregnadoras, cepilladores, finger-jointer, etc. Existe una notoria falta de capacidad de secado, por lo cual será fundamental invertir en este rubro, así como en plantas impregnadoras. Serán también de utilidad los finger-jointers y los cepilladores, considerando, por ejemplo, que se espera un aumento de las ventas de madera corta de Pino radiata, libre de defectos. Por otra parte, la calidad de la madera elaborada en el país es usualmente baja (12): uno de los factores para mejorar dicha calidad sería contar con moldureras, cepilladores y otros equipos de elaboración en buen estado.

g) Otras maquinarias que deberán incrementar su importancia en el país son los astilladores y los descortezadores, debido al exitoso comienzo y al promisorio futuro de la exportación de astillas, actividad que permitirá a los aserraderos obtener una mayor rentabilidad global. Lo anterior conducirá a incrementar las inversiones o reposición de astilladores (13). Además, todo esto puede implicar nuevas inversiones en los puertos para facilitar el manejo, almacenamiento y embarque de las astillas.

h) En relación a las inversiones para las industrias de madera aserrada, se estima que la capacidad instalada actual no será capaz de absorber el enorme recurso forestal en crecimiento, pese a que actualmente existe una gran capacidad ociosa (alrededor del $50 \%$ o). Por una parte, se calcula que para los próximos quince años se requerirán alrededor de 10 a 12 nuevos aserraderos de $100.000 \mathrm{~m}^{3}$ /año en promedio, con sus respectivos secadores y equipos de cepillado; aparte de esto, se estima que para la producción de componentes de muebles serán necesarias 
del orden de 10 plantas, con capacidad de $10.000 \mathrm{~m}^{3} /$ año (1). Por otra parte, de la inversión pronosticada para el sector forestal hasta fines del siglo, que asciende a USS 3.000 millones (3), los expertos (5) señalan que alrededor de un tercio deberá destinarse a nuevos aserraderos y plantas de elaboración o a sus respectivas modernizaciones.

i) finalmente, con respecto a las inversiones, se debe tener presente que la maquinaria en las industrias de aserrío y elaboración se caracteriza por una gran diversidad de marcas. Tal vez se podrían generar ahorros si se orientan las compras a menos marcas y modelos, especialmente en lo que se refiere a la mantención industrial, la cual supuestamente se vería facilitada $(1,5)$. Además, se supone posible lograr una cierta especialización de muchos aserraderos que procesen sólo Pino radiata, aunque también dicha especialización dependerá del producto que se requiera fabricar.

\section{CONCLUSIONES Y RECOMENDACIONES}

A continuación se sintetizan algunas de las conclusiones y recomendaciones que se estiman fundamentales como punto de partida, con el fin de mejorar la calidad y uso de la madera aserrada producida en el pais y el proceso de producción de los aserraderos:

a) Es necesario aplicar adecuadas técnicas de manejo y de silvicultura (raleos, podas, etc.) al bosque destinado para la industria de aserrío. Lo ideal será obtener trozos de mayores diámetros y sin nudos, para lo cual se requerirán raleos y podas tempranas.

b) Para reducir defectos relacionados con la terminación superficial, escuadrias desuniformes y variación de espesores de la madera y para incrementar el rendimiento del proceso (materia prima-producto), será altamente prioritario efectuar una adecuada mantención a las sierras. principalmente un buen tensionado y afilado.

c) En relación a lo anterior, se requiere capacitar personal a todo nivel, principalmente técnicos de alto nivel para trabajar en los aserraderos y especialistas en afilado y mantención de sierras.

d) Es altamente conveniente efectuar una mantención preventiva a la maquinaria, en vez de correctiva. Entre otras cosas, esto alarga la vida útil de los equipos y disminuye costosas interrupciones de operación.

e) El secado artificial de la madera aserrada es en el presente uno de los principales problemas y desafíos si se pretende mejorar la calidad y las exportaciones de este producto. Por una parte, habrá que investigar y corregir diversas deficiencias en relación a las técnicas y variables de secado para las especies de interés nacional. Por otro lado, en Chile existe una urgente necesidad de aumentar significativamente la capacidad de secado artificial. Será fundamental invertir en secadores artificiales y también en plantas impregnadoras.

f) En relación a diversos problemas de operación, una posible solución para los propietarios de bosques o aserraderos chicos sería unirse a fin de construir aserraderos de mayor capacidad, más eficientes, con secadores artifíciales y aptos para producir productos de calidad.

g) Para promover y optimizar el uso y las exportaciones de madera aserrada, será fundamental dictar, exigir y aplicar normas o especificaciones de calidad para el producto. El exigir estas normas es además uno de los factores importantes que se deben considerar si se pretende mejorar técnicamente los aserraderos.

h) Se estima que en los próximos años se requerirá una mayor cantidad de equipos como el chipper canter y las sierras circulares dobles, debido a que, como materia prima, se contará en general con trozos de diámetros más delgados que los actuales.

i) Debido al auge en las exportaciones de astillas y al alto precio que ellas están alcanzando, será necesario invertir en astilladores y descortezadores. 
j) Finalmente, se aprecia que en Chile existe carencia de investigación básica y aplicada tendiente a mejorar en forma importante la calidad de la madera aserrada y la productividad y rendimiento del proceso de aserrio del Pino radiata y de las otras especies comerciales. Faltan datos básicos y de interés práctico para procesar eficientemente las especies. información referente a facilidad de aserrío, desgaste relativo de los elementos de corte, parámetros angulares óptimos de los dientes, consumo de potencia, velocidad de avance, etc. En otras palabras. se requiere obtener datos para la realidad chilena, ya que a menudo se trabaja en forma tentativa con datos obtenidos para especies extranjeras y para realidades diferentes.

\section{REFERENCIAS BIBLIOGRAFICAS}

1. CORPORACION DE FOMENTO DE LA PRODUCCION. 1986. Seminario. Secado industrial de la madera. Apuntes mimeografiados. Concepción, Chile, CORFO. s.p.

2. CHIIE FORESTAL. 1985. Empresarios analizan exportaciones forestales. Santiago, Chile No 122:14-15.

3. CHILE FORESTAL. 1986. Chips a los países escandinavos. Santiago, Chile. No 129:7-8.

4. FUNDACION CHILE, UNIV. DE CHILE. 1983. Diagnóstico de las industrias productoras de elementos de madera. Santiago, Fundación Chile. s.p.

5. FUNDACION CHILE, UNIV. DE CHILE E INSTITUTO FORESTAL. 1983. Desarrollo de las ventajas comparativas de la madera en la construcción de viviendas. Santiago, Fundación Chile. 65 p.

6. INSTITUTO FORESTAL. 1985. Antecedentes sobre el pino insigne y sus industrialización en Chile. Santiago, Chile, INFOR. 64 p.

7. INSTITUTO FORESTAL/CORPORACION DE FOMENTO DE LA PRODUCCION. 1985. Directorio de productores y exportadores forestales 1984. Santiago, Chile, INFOR/CORFO. Serie Intormática No 24. s.p.

8. - 1985. La industria del aserrío durante 1984. Santiago, Chile, INFOR/CORFO. Serie Informática No $25.90 \mathrm{p}$.

9. - - 1985. La industria dimensionada y elaborada de madera en Chile. Santiago, Chile, INFOR/CORFO. Serie Informática № $22.85 \mathrm{p}$.

10.-- - 1986. Estadísticas forestales 1985. Santiago, Chile INFOR/CORFO. Serie Informática No 34. 98 p.

11.-- - 1986. La industria del aserrío durante 1985. Santiago. Chile, INFOR/CORFO. Serie Informática No $35.79 \mathrm{p}$.

12. PEREZ G., V. 1983. Manuel de propiedades físicas y mecánicas de maderas chilenas. Santiago, Chile, CONAF/PNUD/FAO, FO:DP/CHI/76/003. Documento de Trabajo No $47.451 \mathrm{p}$. 